\title{
Robot Structure and Motion Control Design Based on UG and Proteus
}

\author{
Yuhang Sheng \\ Tianjin University of Science and Technology, China \\ E-mail:1346733865@qq.com \\ www.tust.edu.cn
}

\begin{abstract}
The paper is to design a six-degree-of-freedom biped robot by the research on the humanoid characteristics of the biped robot. Our biped robot chooses the steering gear ASMC-03B as its power unit, we restricts the model parameters by analyzing the function relationship between the steering gear torque and the volume, and regulates the size of every parts, and create a motion analysis model. In the hardware part, Arduino UNO, which is used as the main control chip, realize the communication between the main control chip and the servo drive module PAC9685 through the IIC bus protocol, which saves the main control chip resources and ensures the execution efficiency. In the simulation part, it is to simulate the steering angle of the steering gear, and output it in the form of a waveform.
\end{abstract}

Keywords: Keywords: Biped robot,6-degrees of freedom, Mechanical structure, Motion control

\section{Introduction}

With the rapid development of science and technology in today's society, robots are playing an increasingly important role in human life. The invention of robots is one of the greatest inventions in the history of human development. At the same time, it is a very inclusive cross-disciplinary. Biped robot, as one of the hot topics in the field of robot research, has been paid much attention, and its research results are more outstanding than other types of robots.

Relative to domestic, foreign biped robot research field started earlier. Among them, Japan is the first country to invest in biped robot research ${ }^{1}$. The DOF of biped robot has experienced the development from PLANAR DOF ROBOT TO SPATIAL DOF robot, the working mode of biped robot has developed from "teaching-reproduction" to autonomous decision-making robot. Recently, the most interesting biped robot research abroad is the Atlas Robot developed by Boston Power Company. It has a strong perception system and decision-making system, when encountered obstacles to self-judgment to adjust the center of gravity, and by updating the gait to plan the landing site to get away from obstacles. The latest version of Atlas adds body coordination and extreme sports such as cool running and triple jumping on one foot, as shown in Fig.1.

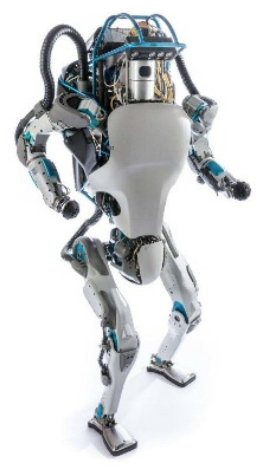

Fig.1. Atlas biped robot

Compared with foreign countries, domestic research on biped robot technology started late. In 1985, “863” plan included the research of intelligent robot in "the outline of China's high-tech Research and Development Program". Among the universities with outstanding achievements in the field of biped robots are the Harbin Institute of Technology, the National University of Defense Technology, and the Beijing Institute of Technology. In recent years, the outstanding achievements in the field of biped robot research in China are the "human-shaped robot Butler" Walker launched by 
Shenzhen Youbixuan Co. , Ltd. in 2019, as shown in Fig.2, its range of motion ability includes all-round free walking, stable climbing and going up and down stairs, the use of three-dimensional visual navigation positioning system to achieve navigation and obstacle avoidance functions, applications such as remote monitoring, education and smart home.

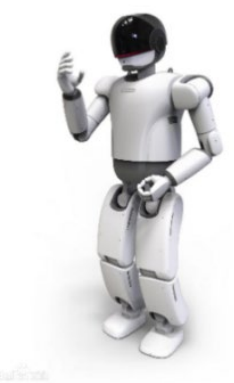

Fig.2. Humanoid Machine Butler

But, the robot product production cycle is long, in the production process unpredictable factor is many. Based on this premise, this paper designs a 6-dof biped robot which meets the requirements by doing the pioneering work to the biped robot model.

\section{Mechanical design}

Before modeling the biped robot, the Mechanical Structure Diagram of the biped robot should be constructed, and then the joint and leg structure should be defined. The mechanical structure of biped robot can be designed according to the anatomy and physiology of human body, the left and right legs are symmetrically distributed, and 6 joints are 2 hip joints, 2 knee joints and 2 ankle joints. The mechanical design of the biped robot is shown in Fig.3.

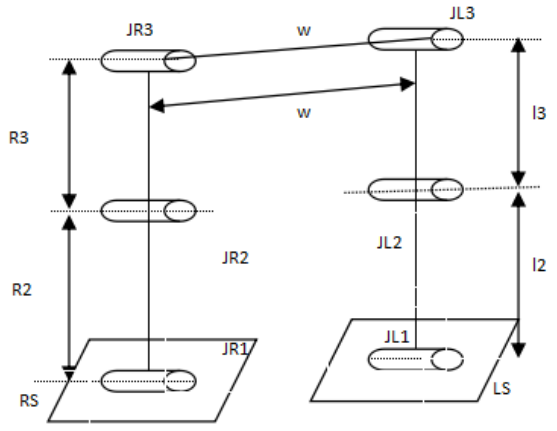

Fig.3. mechanical structure of robot

\section{Design UG model}

Computer aided technology (CAD) is widely used in the field of mechanical design. The application of CAD design combines computer application with engineering design. parametric design is the Yenshin of CAD technology. This article uses the Ug drawing software, which has the powerful compound modelling function, the modularization is more centralized. The mechanical structure design of biped robot can be divided into two parts: actuator selection and parameter design. The steering gear is Asmc-03b, and the Working Torque is $0.38 \mathrm{~N} \cdot \mathrm{M}$. Parameter design ${ }^{2}$ refers to the design method of controlling the size and installation position of the parts through the core parameters, normalizing the size of the parts to a reasonable range. Among them, the working torque of the steering gear is an important index to standardize the size of the parts. According to the physical definition of Torque, the Torque is equal to the cross product of the radial vector of the rotating shaft and the acting force, and the expression of the Torque is as shown in (1).

$$
\mathrm{M}=\mathrm{L} \times \mathrm{F}
$$

Bring $\quad \mathrm{F}=\mathrm{m} \times \mathrm{g}$ and $\rho=m / V$ into available

$$
\rho \times \mathrm{V} \times \mathrm{g} \times \mathrm{L}<0.38 \mathrm{~N} \cdot \mathrm{M}
$$

When the size of the robot is designed, the parameters of the Cross section can be set according to the constraint conditions, and the size is reasonable.

The Mechanical Structure Model of biped robot is composed of steering gear, rudder plate, waist u-shaped support, foot plate, a-shaped parts, leg structure function support, z-shaped function support and other parts. By establishing the connection relation of each part and the constraint relation of the parts, the parts added to the assembly environment can be located precisely. The biped robot model assembled by constraint command is shown in Fig.4.

(C) The 2021 International Conference on Artificial Life and Robotics (ICAROB2021), January 21 to 24, 2021 


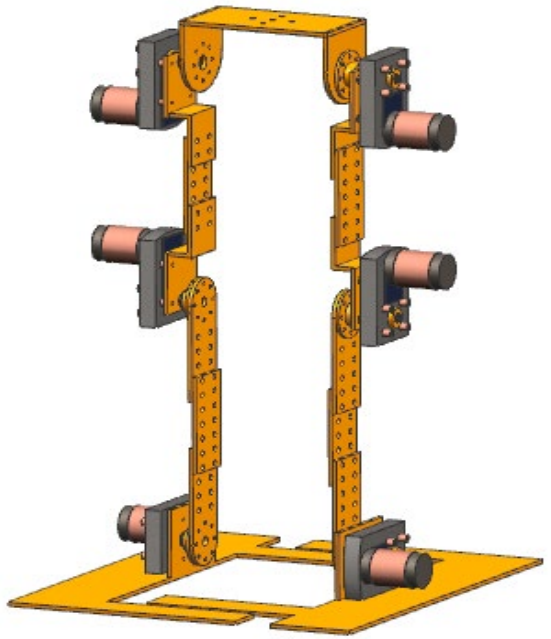

Fig.4. robot assembly drawing

\section{Circuit design of Proteus}

Based on the Proteus simulation circuit software, the hardware circuit of biped robot is designed to drive the steering gear. Proteus circuit design part mainly includes: Power supply voltage-stabilizing circuit, mcu Minimum System Circuit, rudder drive circuit, rudder circuit.

The power supply module is composed of three parts of regulated power supply circuit. The components of each regulated power supply circuit are composed of 78 series three-terminal regulator, capacitor, polar capacitor and power supply terminal. The regulated power supply circuit supplies power to the minimum system circuit, SERVO0, SERVO1, SERVO2, SERVO3, SERVO4 and SERVO5 rudder circuit respectively.

Single-chip microcomputer circuit ${ }^{3}$ is the core of the minimum system circuit of single-chip microcomputer, and is an indispensable part of the design control circuit. The main control chip takes the ATmega $328^{4}$ core as the microprocessor, and completes the tasks of data storage, computation, peripheral device control and interrupt processing.

The SERVO drive circuit uses PAC9685 chip, in which the data transmission pin of SDA and SCL clock pulse pin communicate with the minimum system circuit of single chip microcomputer through IIC bus, and the LED pin is used as the output of PWM wave by connecting the row of pins of 16 pins, A0, A1, A2, A3, A4, A5 are the chip addresses for the driver chips and are connected to the enable signal.
The steering gear circuit is controlled by a micro-controller, which communicates with the steering gear drive module via IIC bus. The steering gear drive module outputs PWM wave to control the steering gear. The hardware circuit diagram is shown in Fig.5.

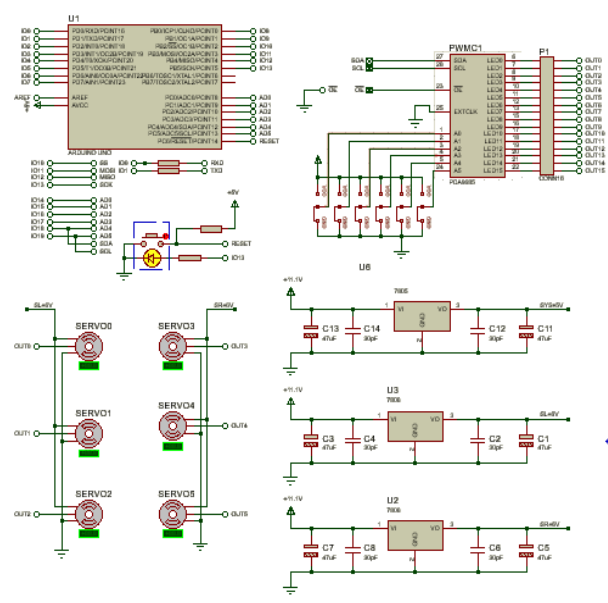

Fig. 5. Hardware circuit

\section{Simulation experiment of Proteus circuit}

The hardware-in-the-loop simulation is illustrated in Fig.6 with an example of a single steering gear experiment. The adjustable potentiometer (a) and the display screen (b) are added to make the circuit simulation visual. By adjusting the potentiometer to output analog voltage, analog voltage signal transmission to the microcontroller minimum circuit, SCM internal voltage value according to register configuration, output the corresponding PWM wave drive actuator rotation angle.

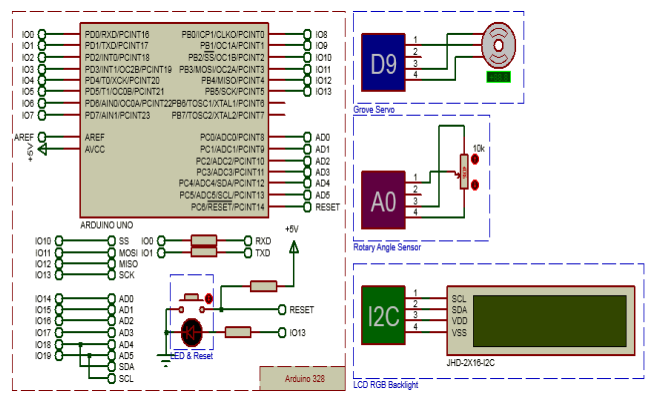

Fig.6. Single steering engine circuit diagram

(C) The 2021 International Conference on Artificial Life and Robotics (ICAROB2021), January 21 to 24, 2021 
By adjusting the adjustable potentiometer to control the pulse width of the steering gear, the Steering Gear Angle from $-90^{\circ}$ to $90^{\circ}$ rotation, as shown in Fig. 7 .

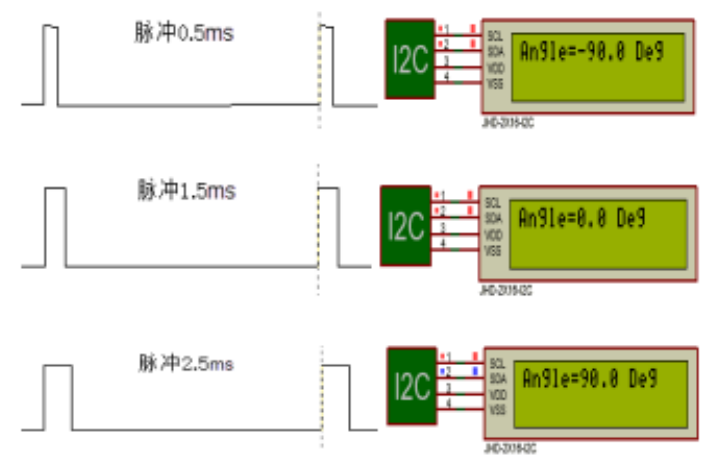

Fig.7. Control diagram of pulse width and angle

The experiment shows that the pulse width is controlled by an adjustable potentiometer, the duty cycle of the actuator is changed from 0 to $100 \%$, and the rotation angle of the actuator is displayed by a display screen, so as to achieve precise control.

\section{Conclusion}

The structure and Motion Control Circuit of biped robot are designed in this paper. In the part of mechanical structure design, through the analysis of the relationship between the rudder torque and material, the dimensions of the parts are standardized to reach a reasonable range. Modeling and assembly of parts using UG software. In the part of Motion Control Circuit, the Proteus circuit is mainly designed, the Arduino Uno is used as the control chip, PAC9685 is used as the actuator module, and 78 series three-terminal voltage regulator is used as the regulator module. In the part of hardware circuit design, the steering gear rotation is simulated by visual programming to realize the precise control of the steering gear.

\section{References}

1. Chen C, Shih B, Shih C, et al. Design, modeling and stability control for an actuated dynamic walking planar bipedal robot. Journal of Vibration and Control, 2013, 19(3): 376-384.

2. Yunqiang Li, Guobin Lin. Design of intelligent parking lot based on Arduino. IOPConference Series: Materials Science and Engineering, 2019, 490(4).

3. Yuan M, Kang M, Ding Y. Parametric Model Of Excavator Working Equipment Based On The Advanced (C) The 2021 International Conference on Artificial Life and Robotics (ICAROB2021), January 21 to 24, 2021
Development Of UG. International Conference on Mechanical Engineering \& Intelligent Systems. 2015.

4. Marwa F Areed. A keyless Entry System based on Arduino board with Wi-Fi technology. Measurement, 2019, 139. 